\title{
OPTIMIZATION AND IN VITRO EVALUATION OF THE RELEASE OF CLASS II DRUG FROM ITS NANOCUBOSOMAL DISPERSION
}

\author{
SADEQ J. AL-SAKINI, NIDHAL K. MARAIE \\ Department of Pharmaceutics, College of Pharmacy, Mustansiriyah University, Baghdad, Iraq \\ Email: sadek.sakini@gmail.com
}

Received: 31 Oct 2018, Revised and Accepted: 09 Jan 2019

\section{ABSTRACT}

Objective: This work involves investigation and evaluation of the factors that affect the preparation and the release of the model class II drug (erythromycin) to optimize the efficiency of its prepared nanocubosomal dispersion to give very fast initial burst effect within the first hour that can continue for further two hours.

Methods: The work involved preparation of ten formulas of cubosomal dispersion by emulsifying different concentrations of glyceryl monooleate (GMO) (lipid content)/surfactant mixtures which were nano-sized and characterized morphologically by Transmission electronic microscopic (TEM), zeta potential, particle size, polydispersity index (pdI), pH, entrapment efficiency, conductivity test, dilution test and in vitro drug release.

Results: The selected nanocubosomal formula (F1) showed pH (7.41), particle size (315.05 nm), pdI (0.194), zeta potential (-30.852), entrapment efficiency (91\%) and gave a 70\% drug release within the first hour of the in vitro test and continued until it gave $96.3 \%$ drug release with further $2 \mathrm{~h}$.

Conclusion: this work succeeded in preparing optimized cubosomal dispersion for erythromycin using different GMO/poloxamer 407 percent. The optimum formula gave an immediate release of the model drug (erythromycin) and it was ready to be incorporated in any suitable dosage form to give fast onset of action.

Keywords: Cubosomes, Erythromycin, GMO, Poloxamer 407, Nanotechnology

(c) 2019 The Authors. Published by Innovare Academic Sciences Pvt Ltd. This is an open access article under the CC BY license (http://creativecommons.org/licenses/by/4.0/) DOI: http://dx.doi.org/10.22159/ijap.2019v11i2.30582

\section{INTRODUCTION}

Development of colloidal carriers as delivery systems for pharmaceutical drugs has stimulated an exponential growth for the encapsulation of bioactive drug molecules into relatively non-toxic and inert carriers for the in vivo delivery constitutes a promising approach for the improvement of their therapeutic index while reducing the side effects [1]. Exceptional success has been made to improve efficacy by developing lipid-based carriers. Cubosomes are structured liquid crystalline particles, composed from certain amphiphilic lipids in definite proportions. It consists of lipid bilayers that are organized in three dimensions as honeycombed structures and divided into two internal aqueous channels that can be used by various bioactive ingredients, such as chemical drugs, peptides and proteins [2]. Due to their unique properties such as thermodynamic stability, bio adhesion, the ability to encapsulate hydrophilic, hydrophobic and amphiphilic substances, and the potential for controlled release through functionalization, cubosomes are regarded as promising vehicles for different routes of administration [3]. Cubosomes are made by the self-assembly of low molecular weight lipids. These lipids are characterized by a maximum continuous interface and high interface to volume ratio, which makes them promising candidates as efficient adsorbents and host- guest applications [4]. The most characterized system of bicontinuous cubic phase forming lipid is the glyceryl monooleate (GMO)/water system. Monoglycerides are non-toxic, biodegradable and biocompatible materials that can self-associate depending on the temperature and aqueous forming content. In this work a model drug (erythromycin) was used which is used to treat bacteria responsible of the skin and upper respiratory tract infections, including Staphylococcus, Streptococcus, Corynebacterium and Haemophilus genera [5]. The aim of this work was to optimize the cubosomal dispersion of the model drug through studying different variables including using different ratios GMO: Smix, type and time of stirring to improve loading capacity and by enhancing penetration of the drug through skin barrier and improving its effectiveness. The release of the drug that may improve patient compliance through its quick relieve of different bacterial infections.

\section{MATERIALS AND METHODS}

\section{Materials}

Erythromycin powder and poloxamer 407 was purchased from SigmaAldrich, Chemie GMBH, Germany, Glyceryl mono oleate was purchased from Hangzhou Hyper chemicals, China, Potassium phosphate monobasic and Sodium hydroxide were purchased from Himedia, India.

Table 1: Composition of the prepared nano cubosomal dispersion formulas

\begin{tabular}{|c|c|c|c|c|}
\hline Formula code & GMO & Poloxamer 407 & Drug & Buffer \\
\hline F1 & $4.4 \%$ & $0.6 \%$ & $2 \%$ & q. $\mathrm{s}$ \\
\hline $\mathrm{F} 2$ & $4.2 \%$ & $0.8 \%$ & $2 \%$ & q. $s$ \\
\hline F3 & $4.0 \%$ & $1.0 \%$ & $2 \%$ & q. $s$ \\
\hline F4 & $3.8 \%$ & $1.2 \%$ & $2 \%$ & q. $s$ \\
\hline F5 & $3.6 \%$ & $1.4 \%$ & $2 \%$ & q. $s$ \\
\hline F6 & $3.4 \%$ & $1.6 \%$ & $2 \%$ & q. $s$ \\
\hline F7 & $3.2 \%$ & $1.8 \%$ & $2 \%$ & q. $s$ \\
\hline F8 & $3.0 \%$ & $2.0 \%$ & $2 \%$ & q. $s$ \\
\hline F9 & $2.8 \%$ & $2.2 \%$ & $2 \%$ & q. $s$ \\
\hline F10 & $2.6 \%$ & $2.4 \%$ & $2 \%$ & q. $s$ \\
\hline
\end{tabular}




\section{Preparation of cubosomal dispersion formulas}

Preparation of cubosome dispersions formulas was done by emulsification of Glyceryl mono oleate (GMO)/surfactant mixtures. Poloxamer 407 was used as the nonionic surfactant in a concentration range between $0 \%$ and $16 \% \mathrm{w} / \mathrm{w}$. The concentration of the GMO/Poloxamer mixture was $5 \% \mathrm{w} / \mathrm{w}$ with respect to the weight of the dispersion (table 1). Erythromycin concentration used was 2\%. GMO and Poloxamer 407 were melted on a hot plate and then erythromycin was dispersed in the molten mixture. The molten mixture was then added drop by drop to the aqueous phase (phosphate buffer 7.4) at $70^{\circ}$ $\mathrm{C}$ under mechanical stirring at $1500 \mathrm{rpm}$. The dispersions were maintained under mechanical stirring and they were cooled to room temperature to solidify the lipid droplets. The dispersions were sonicated for $10 \mathrm{~min}$ using probe ultrasonicator at $300 \mathrm{w}$ in order to reduce the droplet size to the nanoscale then they were stored in glass bottles at room temperature $\left(25^{\circ} \mathrm{C}\right)$ for further investigations $[6,7]$.

\section{The physical appearance of the formulas}

Evaluation of the physical properties (color and homogeneity) for all the prepared nanocubosomal dispersion formulas (F1-F10) was done visually.

\section{pH determination of the formulas}

The $\mathrm{pH}$ of the formulations (F1-F10) was determined using a digital $\mathrm{pH}$ meter by immersing the electrode of the device in a beaker containing $10 \mathrm{ml}$ of each of the nano cubosomal dispersion formulas and record the results after two minutes.

\section{Zeta potential, particle size and polydispersity index} determination

Measurement of the mean particle size (mean diameter), zeta potential (particle surface charge) and polydispersity index (size range of particles) was done by using dynamic light scattering method (ZetaPlus Particle Sizing), by this technique the light scattering fluctuations were examined, this fluctuation is due to the Brownian motion of cubosomal dispersion particles. It measures the size accurately in the range of 0.3 $\mathrm{nm}$ to $10 \mu \mathrm{m}$. One $\mathrm{ml}$ of the diluted cubosomal dispersion was injected into folded capillary zeta cell and monitor the light scattering at $25^{\circ} \mathrm{C}$ $\left(190^{\circ}\right.$ angle). The average particle size, polydispersity index, and zeta potential values for (F1-F10) were recorded [8].

\section{Separation test}

This test is very important to check the physical stability of the dispersions. The centrifugation of each nanocubosomal dispersion formula (F1-F10) for $30 \mathrm{~min}$ at $(10,000 \mathrm{rpm})$ was applied to check the nanocubosomal dispersions resistance for separation [8].

\section{Drug content}

Accurately, one ml of each nano cubosomal dispersion formula (F1F10) (containing $20 \mathrm{mg} / \mathrm{ml}$ erythromycin) was transferred to a volumetric flask $(100 \mathrm{ml})$ and $70 \mathrm{ml}$ methanol was appended, and the clear solution was achieved after sonication for $30 \mathrm{~min}$. The solution volume was diluted with methanol to $100 \mathrm{ml}$ and subjected to centrifugation for $15 \mathrm{~min}$ at $3000 \mathrm{rpm}$ then filtered using Millipore filter $0.22 \mu \mathrm{m}$. The content of erythromycin was determined spectrophotometrically using the UV-Visible spectrophotometer (Shimadzu 1650 PC-Japan) at $\lambda \max 285 \mathrm{~nm}$ [9].

\section{Entrapment efficiency}

For the determination of loading capacity (entrapment efficiency), each of the cubosomal dispersions (F1-F10) were subjected for centrifugation at $15000 \mathrm{rpm}$ for a period of $30 \mathrm{~min}$, and the supernatant liquid was collected, diluted appropriately and estimated using UV visible spectrophotometer at $\lambda$ max $285 \mathrm{~nm}$ [10]. The percent of entrapment efficiency (\%EE) was calculated by the following equation:

$$
\% \mathrm{EE}=\frac{\text { total drug }- \text { free drug }}{\text { total drug }} \times 100
$$

\section{Conductivity test}

Electrical conductivity measurement was done to determine the nature of the prepared nanocubosomal dispersion formulas (F1-
F10). If the external (continuous) phase is aqueous, the nanocubosomal dispersions are o/w (high conduction) but if the internal (dispersal) phase is aqueous, the nanocubosomal dispersions are w/o (no conduction). Measuring of electrical conductivity $(\sigma)$ was made by the use of a conductometer via immersing the conductometer probe in $10 \mathrm{ml}$ of the prepared dispersion in a beaker at room temperature and the apparatus will register the results in $\mu \mathrm{s} / \mathrm{cm}$ [11].

\section{Dilution test}

The dilution test was done to check the physical stability of nanocubosomal dispersion formulas (F1-F10). The aqueous dilution test was carried out by diluting $1 \mathrm{ml}$ of each nanocubosomal dispersion formula to $50 \mathrm{ml}, 100 \mathrm{ml}$, and $500 \mathrm{ml}$ with double distilled water at $37^{\circ} \mathrm{C}$ with constant stirring at a speed of $50 \mathrm{rpm}$ and observed visually the clarity, turbidity, cracking and phase separation [12].

\section{TEM study}

The nano cubosomal dispersions were further characterized by TEM operating at 30 Kilovolts. A drop of the diluted formula was allowed to be deposited on the circular copper film grid of 300 mesh and then stained with formvar and left for drying. The size and shape of the cubosomes were measured after the complete drying of the slide by observing the slide under the microscope [13].

\section{In vitro release}

The release of erythromycin from the nano cubosomal dispersion formulas (F1-F10) was done by using dialysis membrane (MWCO $2000 \mathrm{Da}$ ). Rotating paddle dissolution apparatus type II was used to measure the in vitro drug release from all prepared formulas. The sealed dialysis bag containing nanocubosomal dispersion formula (equivalent to $120 \mathrm{mg}$ erythromycin) was sunken in $500 \mathrm{ml}$ phosphate buffered ( $\mathrm{pH} 7.4$ dissolution media) with a speed of 50 $\mathrm{rpm}$. The temperature of the medium was maintained at $37 \pm 0.5^{\circ} \mathrm{C}$. Five $\mathrm{ml}$ aliquots outgoing for suitable time periods and immediately making the replacement with fresh dissolution medium. The drug content in the withdrawn sample was determined spectrophotometrically by using a UV-Vis spectrophotometer at the selected $\lambda \max 285$ [14].

\section{FTIR study}

The FTIR spectrum of the dispersions in comparison with the pure drug was recorded by the use of an FT-IR spectrophotometer. They were obtained using liquid cell by dripping several drops of the sample onto $\mathrm{NaCl}$ or $\mathrm{KBr}$ aperture plate and then sandwiching it under another aperture plate so that no gas bubbles are trapped. The thickness can be adjusted according to the sample absorbance by appropriately tightening the screws or by inserting spacers between the aperture plates [15].

\section{RESULTS AND DISCUSSION}

\section{Physical appearance}

All the formulas (F1-F10) appeared as a white homogenous milky solution without any aggregates.

\section{pH determination}

The $\mathrm{pH}$ of the nano cubosomes formulations (F1-F10) was measured using $\mathrm{pH}$ meter. The $\mathrm{pH}$ values were ranged (7.29-7.42), and this matches the skin requirements for topical preparations to avoid skin irritation and agreed with the results obtained with topical cubosomal gel for ketoconazole [17].

\section{Zeta potential, particle size, and PDI determination}

Particle size measurement was carried out to confirm that all particles of the dispersion are in the nanometer size range (fig. 1). The effective diameter of all cubosomal dispersions was in the nanometer range (average particle size values ranged from $315 \mathrm{~nm}$ to $416 \mathrm{~nm}$ ), with a polydispersity index of $<1$, the low value of polydispersity index $0.08-0.7$ is considered to be desirable for uniform distribution and homogeneity of nano-sized particles within the preparation. While pdI value $>0.7$ to less than 1 is considered to 
have a broad distribution of particle size [18]. The zeta potential of all formulas were comparably low, ranging between $(-24.786$ to$30.852 \mathrm{mV}$ ), which could be due to the absence of charge in cubosomal dispersion ingredients, this is mainly because of the use of non-ionic surfactant (poloxamer 407) and the presence of fatty acid (oleic acid oil) which generally made the surface charge of the particle negative [19], the effect of zeta potential on the stability of nanoparticle was explained by rule of thumb. This rule states that values of zeta potential in the range $(\leq-30 \mathrm{mV})$ to $(\geq+30 \mathrm{mV})$ indicate that there are good stability and values in the range $(\leq-60 \mathrm{mV})$ to $(\geq+60 \mathrm{mV})$ indicate that there is excellent stability in the formulation [20]. For nonionic surfactant (poloxamer 407) that act by steric stabilization, the value of zeta potential of $20 \mathrm{mV}$ or even lower provided an efficient stabilization due to that the non-ionic surfactant (poloxamer 407) provided good steric stability for maintaining the stability of single layer nanodispersions [21].



Fig. 1: Particle size distribution by number of the selected formula (F1)

\section{Separation test}

No sedimentation, creaming or phase separation upon centrifugation for all the prepared nano cubosomal dispersion formulas (F1-F10) was observed, this is a good indication of their high stability [22]. This proved that the thermal motion of the dispersion droplets (the Brownian motion) exceeded the external forces such as centrifugation and/or gravitation [23].

\section{Drug content}

The drug content of all nano cubosomal dispersion formulas (F1-F10) results are inconsistent with the requirements of the USP(16), indicating high adequacy of the preparation method and high content uniformity of the prepared formulas, where all the prepared nano cubosomal dispersions have a drug content between (90-98\%) and this agreed with the acceptable range according to the USP (85-115\%) [24].

\section{Entrapment efficiency}

The entrapment efficiency of erythromycin nano cubosomal dispersion formulas is shown in fig. (2). The high entrapment efficiency indicates that the technique applied in the preparation of nanoparticles is applicable and reliable. The entrapment efficiency of (F1-F5) is higher than the entrapment efficiency of (F6-F10) due to the presence of high percent of lipid content (GMO), and as the percent of lipid (GMO) was decreased the entrapment efficiency decreased too, this is mainly due to the high solubility of the drug (erythromycin) in the lipid part of the dispersion. This agreed with Etoposide-loaded nanoparticles made from glyceride lipids [25].

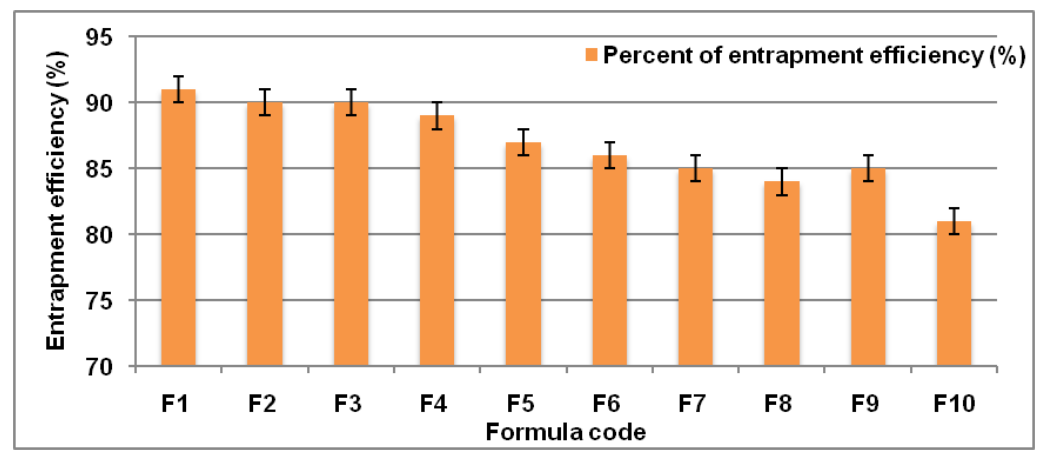

Fig. 2: Entrapment efficiency of erythromycin nano cubosomal dispersion formulas, (values are mean \pm SD) $(n=3)$

\section{Conductivity test}

The electrical conductivity $(\sigma)$ of nanocubosomal dispersions defined as a measurement of materials ability to confirm the transport of an electric charge and it was measured to determine the nature or type of the external phase of dispersion and to detect the phase inversion phenomena. Conductivity measurement depends on the higher conductivity of the water compared to the oil and gives 
high values in o/w dispersions where water is the external phase The results indicated that all the prepared nanocubosomal dispersion formulas were o/w type since high conductivity (76$252 \mu \mathrm{s} / \mathrm{cm}$ ). The higher conductivity is due to a large percentage of water, which allows higher freedom for mobility of ions [26].

\section{Dilution test}

The dilution test is very important to define whether the dispersion is $\mathrm{o} / \mathrm{w}$ or $\mathrm{w} / \mathrm{o}$. If the emulsion is $\mathrm{o} / \mathrm{w}$ type then the addition of more continuous phase (water) will not cause cracking or separation of that emulsion. The results showed that the addition of water to all nanocubosomal dispersion formulas (F1F10) showed clear nanocubosomal dispersion in less than 1 minute with no cracking or separation adding a further indication that they are all o/w type [27].

\section{TEM study}

The shape and size of the nano cubosomal dispersions were characterized by the use of transmission electron microscopy (TEM) as in fig. (3). The images showed cubosomes as dark globules with bright surrounding with average droplet size $(375 \mathrm{~nm})$ and similar shape was obtained with transdermal cubosomes of Etodolac [28].

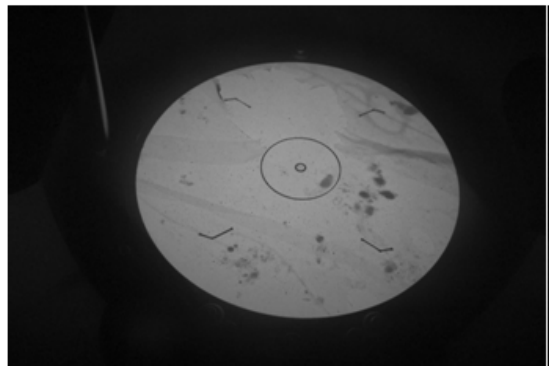

(A)

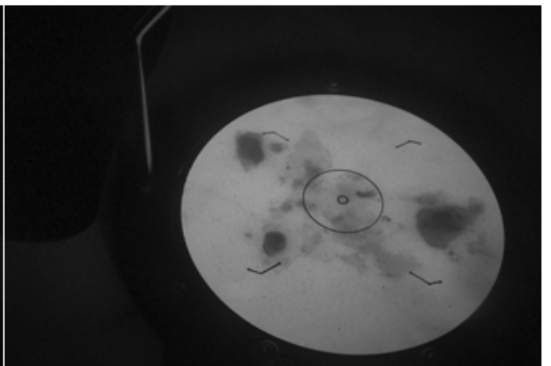

(B)

Fig. 3: (A) TEM picture at zoom 64000 pixels, (B) TEM picture at zoom 180000 pixels

\section{In vitro dissolution test}

The cumulative percentage of erythromycin at different time intervals for each nano cubosomal dispersion is shown in fig. (4). The result of cumulative drug release showed that different GMO content had a profound effect on drug release. The result showed a significant decrease in the initial drug release (after $1 \mathrm{~h}$.) between F1 and F10, 70\% and 45\% respectively. Also, the release after $3 \mathrm{~h}$ was significantly greater for F1 than $\mathrm{F} 10,96 \%$ and $89 \%$ respectively. As the concentration changed from the higher concentration of GMO in F1 (4.4\%) to the lower concentration of GMO in F10 (2.6\%). In addition to that, the concentration of poloxamer 407 in F10 is much higher than F1 and that caused a significant decrease in the release of the drug from F10 in comparison to $\mathrm{F} 1$, since this will be fewer water channels resulting for reinforcement of micellar structure $[29,30]$. The result showed that F1 gave $70 \%$ drug release within $1 \mathrm{~h}$ and the release increasingly continued for the next $2 \mathrm{~h}(96.3 \%)$, which was better than other formulas, and this could be due to the presence of high amount of GMO in F1 (4.4\%) [31].

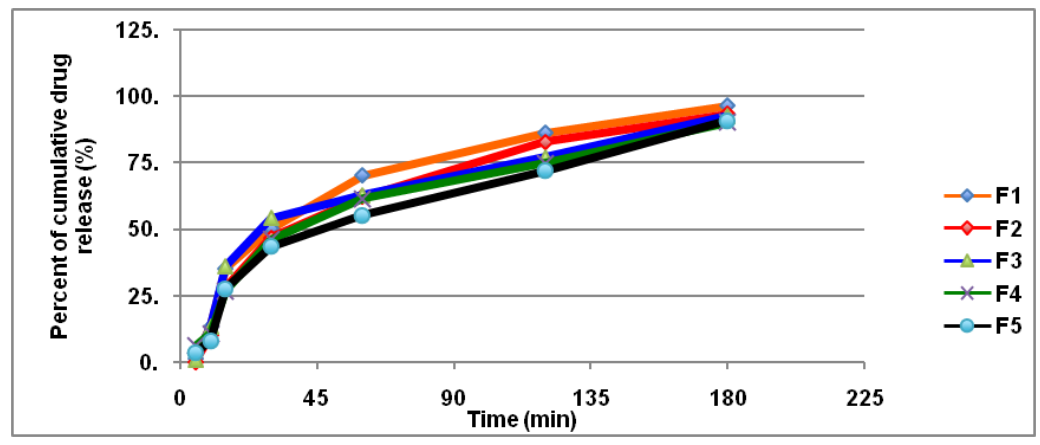

(A)



(B)

Fig. 4: (A) In vitro release profile of erythromycin from (F1-F5) formulas (values are mean \pm SD) (n=3), in phosphate buffer solution (pH 7.4) at $37 \pm 1^{\circ} \mathrm{C}$, (B) In vitro release profile of erythromycin from F6-F10 formulas (values are mean $\left.\pm \mathrm{SD}\right)(\mathrm{n}=3)$, in phosphate buffer solution (pH 7.4) at $37 \pm 1^{\circ} \mathrm{C}$ 


\section{FT-IR study}

The FT-IR spectrum of the pure drug showed the distinctive peaks values, which are $1639 \mathrm{~cm}-1 \mathrm{C}=0$ (carbonyl stretching), $3402 \mathrm{~cm}-1$ $\mathrm{C}-\mathrm{OH}$ (alcohol stretching) and $1085 \mathrm{~cm}-1 \mathrm{C}-\mathrm{O}-\mathrm{C}$ (ether stretching). The FTIR spectrum of erythromycin nanocubosomal dispersion displayed same functional groups band with very slight shifting indicating excellent compatibility of excipients with the drug [32].

\section{CONCLUSION}

Depending on the results obtained in this study, this work revealed that slight change in lipid: surfactant ratio as well as using alternative stirring type and time in comparison to related preparation led to improving entrapment efficiency and gave immediate release of the drug from the prepared emulsions as well as the unique structure of the prepared cubosomes that can improve the antibacterial activity of the model drug and can give quick significant action that will improve patient compliance.

\section{ACKNOWLEDGMENT}

The Authors would like to thank Mustansiriyah University

(www.uomustansiriyh.edu.iq) Baghdad-Iraq for its support in the present work.

\section{AUTHORS CONTRIBUTIONS}

All the authors have contributed equally in the conceptualization and execution of the article

\section{CONFLICT OF INTERESTS}

The authors declared that they have no conflicts of interest

\section{REFERENCES}

1. Date A, Naik B, Nagarsenker MS. Novel drug delivery systems: potential in improving topical delivery of antiacne agents. Skin Pharma Physio 2005;19:2-16.

2. Sherif S, Bendas ER, Badawy S. The clinical efficacy of cosmeceutical application of liquid crystalline nanostructured dispersions of alpha lipoic acid as anti-wrinkle. Eur J Pharm Biopharm 2014;86:251-9.

3. Anbarasan B, Fatima GX, Shanmuganathan S. An overview of cubosomes-smart drug delivery system. Sri Rama J Med 2015;8:1-4.

4. He H. Cubosomes from hierarchical self-assembly of poly (ionic liquid) block copolymers. Nat Commun 2017;8:1-8.

5. Gaynor M, Mankin AS. Macrolide antibiotics: binding site, mechanism of action, resistance. Curr Top Med Chem 2003;3:949-61.

6. Morsi NM, Abdelbary GA, Ahmed MA. Silver sulfadiazine based cubosome hydrogels for the topical treatment of burns: development and in vitro/in vivo characterization. Eur J Pharma BioPharma 2014;86:178-89.

7. Sana K, Poorva J, Sourabh J, Richa Jain, Saurabh B, Aakanchha J. Topical delivery of erythromycin through cubosomes for acne. Pharma Nanotech 2018;6:38-47.

8. Ghosh V, Mukherjee A, Chandrasekaran N. Ultrasonic emulsification of food-grade nanoemulsion formulation and evaluation of its bactericidal activity. Ultrasonics Sonochem 2013;20:338-44.

9. Shweta K, Ganesh K, Preeti K. Development and in vitro characterization of the ocular insert containing erythromycin. Int Res J Pharma 2012;3:246-50.

10. Nasra M, Ghoraba MK, Abdelazem A. In vitro and in vivo evaluation of cubosomes containing 5-fluorouracil for liver targeting. Acta Pharma Sinica 2015;5:79-88.

11. McClements D. Colloidal basis of emulsion color. Curr Opin Colloid Interface Sci 2002;7:451-5.
12. Beg S, Jenaa SS, Patraa CN, Rizwanb M, Swaina S, Sruti J, et al. Development of solid self-nano emulsifying granules (SSNEGs) of ondansetron hydrochloride with enhanced bioavailability of potential colloids and surfaces. Biointerfaces 2013;101:414-23.

13. Setya S, Negi P, Razdan D, Talegaonkar DS. Design, development and in vitro investigation of water in oil nanoemulsion for transdermal delivery. World J Pharma Pharmaceutical Sci 2014;3:1495-512.

14. Ravi PR, Aditya N, Patil S, Cherian L. Nasal in-situ gels for delivery of rasagiline mesylate: improvement in bioavailability and brain localization. Informa Healthcare 2015;22:903-99.

15. Skoog DA, West DM, Holler FJ, Stanley RC. Fundamentals of analytical chemistry. 9th edition. Thomson-Brooks/Cole, USA; 2014;9:658-64.

16. Bachhav JK, Bhairav BA, Saudagar RB. Formulation and evaluation of topical emulgel of ketoconazole by cubosomal technique. World J Pharma Res 2017;6:567-88.

17. Danaei M, Dehghankhold M, Ataei S, Hasanzadeh F, Javanmard $\mathrm{R}$, Dokhani $\mathrm{A}$, et al. Impact of particle size and polydispersity index on the clinical applications of lipidic nanocarrier systems. Pharmaceutics 2018;10:1-17.

18. Daware SU, Saudagar RB. Formulation and development of cubosomes loaded emulgel of fluconazole. Eur J Bio Med Pharma Sci 2017:4:321-31.

19. Rainer H. Zeta potential and particle loading in laboratory practice: an introduction to theory, practical measurement performance, data interpretation. 24th ed. scientific publishing company; 1996.

20. Hoeller S, Sperge A, Valenta C. Lecithin based nanoemulsions: a comparative study of the influence of non-ionic surfactants and the cationic phytosphingosine on physicochemical behavior and skin permeation. Int J Pharm 2009;370:181-6.

21. Tadros TF. Emulsion formation and stability. 1st ed. Germany: Wiley-VCH Verlag Gmb H and Co. KGaA; 2013.

22. Chhabra G, Chuttani K, Mishra AK, Pathak K. Design and development of nanoemulsion drug delivery system of amlodipine besilate for improvement of oral bioavailability. Drug Dev Pharm 2011;37:907-16.

23. USP 32. NF27UP. USA, Rockville MD: The United State pharmacopeial convention Inc; 2008.

24. Harivardhan LR, Murthy RS. Etoposide-loaded nanoparticles made from glyceride lipids: formulation, characterization, in vitro drug release, and stability evaluation. AAPS Pharma Sci Tech 2005;6:158-66.

25. Kalra R. Development and characterization of nanoemulsion formulations for transdermal delivery of aceclofenac. Int J Drug Form Res 2010;1:359-86.

26. Almajidi YQ, Zainab HM, Maraie NK. Preparation and in vitro evaluation of montelukast sodium oral nanoemulsion. Int J Appl Pharm 2018;10:49-53.

27. Salwa S, Azza A, Amany OK. Etodolac transdermal cubosomes for the treatment of rheumatoid arthritis: ex vivo permeation and in vivo pharmacokinetic studies. Drug Delivery 2017;24:846-56.

28. Maraie NK, Almajidi YQ. Effect of different mucoadhesive polymers on the release of ondansetron $\mathrm{HCl}$ from intranasal mucoadhesive in situ gel. Afr J Plant Sci 2017;17:76-84.

29. Wesam RK, Shohei S, Ichiro H, Hiroaki T, Kenji S. A novel chemical enhancer approach for transdermal drug delivery with C17 monoglycerol ester liquid crystal forming lipid. J Oleo Sci 2017;66:443-54.

30. Thakkar V, Korat V, Baldaniya L, Gohel M, Gandhi T, Patel N. Development and characterization of the novel hydrogel containing an antimicrobial drug for treatment of burns. Int J Pharma Invest 2018;6:158-68.

31. Riccia EJ, Lunardi LO, Nanclares DMA, Marchetti JM. Sustained release of lidocaine from poloxamer 407 gels. Int J Pharm 2005;288:235-44.

32. Shimadzu corporation analytical Instruments Division. Shimadzu reference manual. Kyoto, Japan; 2001. 